\title{
Carcinome épidermoïde de l'urètre masculin révélé par une rupture spontanée de l'urètre
}

\author{
Jilani Ghorbel, MD; Ghassen Hafsia, MD; Amine Derouiche, MD; Anis Jrad, MD; Mohamed Chebil, MD
}

Cite as: Can Urol Assoc J 2011;5(3):e36-e39; D01:10.5489/cuaj.09174

Service d'urologie, Hôpital Charles Nicolle, Tunis, Tunisie

\section{Résumé}

Le carcinome épidermoïde de l'urètre masculin est une tumeur rare, les tumeurs de l'urètre tous types confondus représentant moins de $1 \%$ des tumeurs de l'appareil urinaire. Le pronostic reste défavorable malgré un traitement chirurgical énergique. La radiochimiothérapie semble être un traitement prometteur, mais son rôle doit être défini par d'autres études.

Nous rapportons un cas rare de carcinome épidermoïde de I'urètre bulbo-membraneux découvert à un stade localement avancé après observation d'une rupture urétrale transtumorale chez un homme âgé de 70 ans. Le patient a été traité, après drainage vésical, par une irradiation externe associée à une chimiothérapie par cisplatine, et est décédé après progression de la maladie sur un an.

La rupture spontanée de l'urètre transtumorale est un mode de découverte exceptionnel témoignant d'une évolution locale défavorable, ce qui rend ces tumeurs difficilement opérables. Cependant, I'espoir actuel réside dans des protocoles thérapeutiques associant radiothérapie et chimiothérapie.

\section{Introduction}

Le carcinome primitif de l'urètre est une tumeur rare, représentant moins de $1 \%$ de toutes les tumeurs urologiques malignes de I'homme ${ }^{1,2}$. Il prédomine chez la femme par rapport à l'homme à raison d'un ratio de 3:1.

Sa localisation principale chez I'homme est l'urètre bulbo-membraneux (2/3 des cas). Le type histologique le plus fréquent est le carcinome épidermoïde (80\% des cas). Le diagnostic de ces tumeurs est en général délicat car la symptomatologie est peu spécifique et dominée par la présence $d^{\prime}$ une masse palpable sur le trajet de l'urètre et l'existence $d^{\prime}$ un syndrome urinaire obstructif. Son traitement est essentiellement chirurgical mais souvent mutilant. L'espoir actuel réside dans l'association d'une radiothérapie et d'une chimiothérapie mais le pronostic reste défavorable dans la majorité des $\operatorname{cas}^{3}$. En parallèle à la description d'un cas de cancer épidermoïde de l'urètre chez l'homme révélé par une rupture de l'urètre membraneux, nous présentons une revue de la littérature et discutons des aspects épidémiologiques, cliniques et thérapeutiques de cette pathologie.

\section{Observation}

M. D. M., 73 ans, a de longs antécédents de dysurie et d'urétrorragie intermittente. On observe à l'urétrocystographie rétrograde la présence d'une image irrégulière marécageuse de l'urètre bulbo-membraneux (figure 1) en rapport avec des bourgeons tumoraux bulbaires obstructifs objectivés à I'urétéroscopie, réséqués à plusieurs reprises et correspondant tous à des papillomes urétraux. Ce patient a été admis au Service d'urologie pour rétention urinaire aiguë avec urinome sous-cutané infecté s'étendant des régions périnéoscrotale et inguinale gauches jusqu'à la fosse lombaire gauche. Ce tableau clinique a nécessité une large mise à plat et la mise en place d'une cystostomie avec découverte peropératoire d'une masse tumorale indurée au niveau de I'urètre bulbo-membraneux blanchâtre faisant $4 \mathrm{~cm}$, entourée de tissu inflammatoire, nécrosée en son centre avec issue d'urine à travers cette tumeur dont on a fait la biopsie (figures 2 et 3). L'évolution clinique et biologique était favorable sous antibiothérapie. L'examen clinique démontrait une tuméfaction indurée située sur le raphé médian au niveau de l'angle périnéoscrotal. Le toucher rectal démontrait une prostate plate de consistance normale et un plancher vésical souple. De plus, on palpait des adénopathies inguinales droites, fermes et fixées. L'étude histologique des biopsies urétrales concluait à un carcinome épidermoïde bien différencié. Dans le cadre du bilan d'extension, une échographie périnéale et endorectale a été demandée afin d'évaluer l'étendue en profondeur. Elle montrait une tumeur de l'urètre membraneux étendue à la partie postérieure des corps caverneux sans prolongement vers le complexe vésico-prostatique (figure 4). La tomodensitométrie pelvienne montrait qu'il s'agissait d'une tumeur étendue avec des adénopathies inguinales bilatérales infracentimétriques (figure 5). Par ail- 


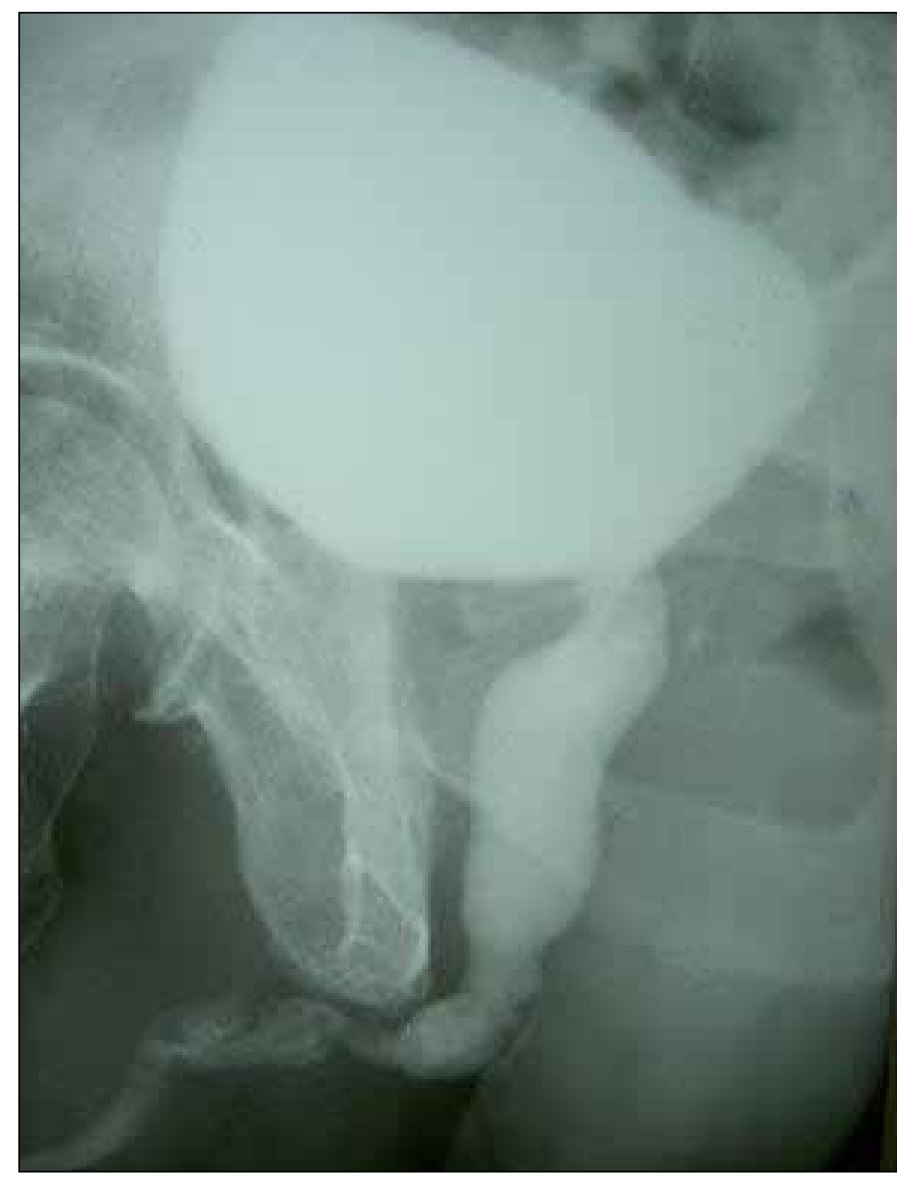

Fig. 1. UCRM: Image irrégulière marécageuse de l'urètre bulbo-membraneux.

leurs, aucune métastase pulmonaire ou hépatique n'a été observée. L'atteinte du corps caverneux gauche classifiait cette tumeur T3, N+, M0 (classification TNM) (tableau 1). Le patient s'est vu prescrire une radiochimiothérapie concomitante en raison de l'évolution locale de la tumeur. II a eu 3 séances hebdomadaires de chimiothérapie à base de cisplatine et 5-FU suivies de 2 séances de radiothérapie. L'évolution était assez encourageante, marquée par une amélioration nette de l'état général et une régression clinique de la masse, ce qui a motivé la poursuite du protocole. Cependant, l'issue a été fatale après une année.

\section{Discussion}

Le cancer primitif de l'urètre masculin est rare. Six cents cas atteints d'une tumeur de l'urètre masculin ont été relevés dans la littérature ${ }^{3}$. L'âge moyen de survenue est de 60 ans ${ }^{4}$. Pour notre part, nous rapportons le seul cas observé qui s'est manifesté par une rupture spontanée transtumorale de l'urètre.

L'étiologie exacte de ce cancer reste encore inconnue. Certains facteurs favorisants ont été proposés. Ces derniers ont en commun un potentiel irritatif : antécédents de sténose

\begin{tabular}{ll} 
Tableau 1. Classification TNM 1997 des tumeurs de l'urètre \\
\hline Ta & Carcinome papillaire non invasif, polypoïde ou verruquex \\
\hline Tis & Carinome in situ \\
\hline T1 & Invasion sous muquese \\
\hline T2 & $\begin{array}{l}\text { Invasion du corps spongieuz ou de la prostate ou du } \\
\text { muscle péri-urétral }\end{array}$ \\
\hline T3 & $\begin{array}{l}\text { Invasion du corps caverneux, de la capsule prostatique, } \\
\text { ou du vagin ou du col vésical }\end{array}$ \\
\hline T4 & Invasion des autres organes de voisinage \\
\hline
\end{tabular}

urétrale, notée chez 25 à $75 \%$ des patients, antécédents de maladie vénérienne ${ }^{4}$, processus infectieux chronique; infection à papillomavirus ${ }^{5}$, traumatisme urétral, urétroplastie ${ }^{6}$; antécédents de polypes urétraux et lésion leucoplasique préexistante qui pourraient agir comme point de départ de la dégénérescence secondaire.

Il semble que dans le cas qui nous concerne, l'association avec le papillome de l'urètre qui est connu comme lésion bénigne pourrait en fait correspondre à une lésion précancéreuse. Ceci reste toutefois à confirmer.

Les deux tiers environ des tumeurs de l'urètre siègent au niveau de l'urètre bulbo-membraneux ${ }^{7}$. Plus rarement au niveau de l'urètre pénien, elles sont en revanche exceptionnelles au niveau de l'urètre prostatique'.

$\mathrm{D}^{\prime}$ un point de vue carcinologique, on considère comme distales les tumeurs de l'urètre pendulaire et comme proximales les tumeurs de l'urètre bulbo-membraneux ${ }^{8}$. Les stades T2 et inférieurs sont considérés comme de faibles stades, alors que les stades T3 et T4 sont des stades élevés ${ }^{8}$.

Sur le plan clinique, les symptômes sont sans spécificité et considérés à tort comme ceux d'un rétrécissement banal, $\mathrm{d}^{\prime}$ 'où des diagnostics posés trop tardivement ${ }^{9}$. Il faut rappeler la nécessité de procéder à des examens complémentaires afin de ne pas retarder le diagnostic de cancer de l'urètre

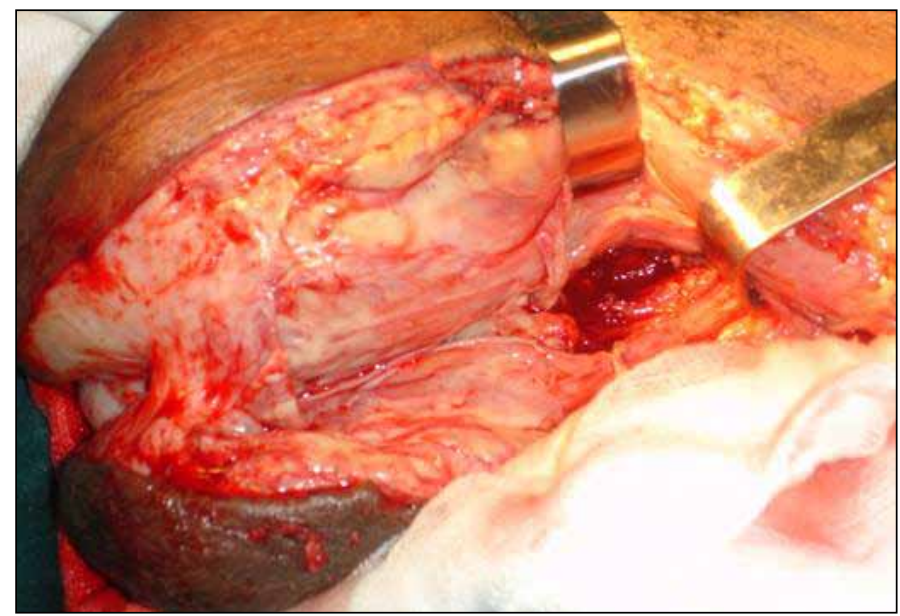

Fig. 2. Découverte peropératoire d'une masse tumorale blanchâtre au niveau de l'urètre bulbo-membraneux, entourée de tissu inflammatoire et nécrosée en son centre. 


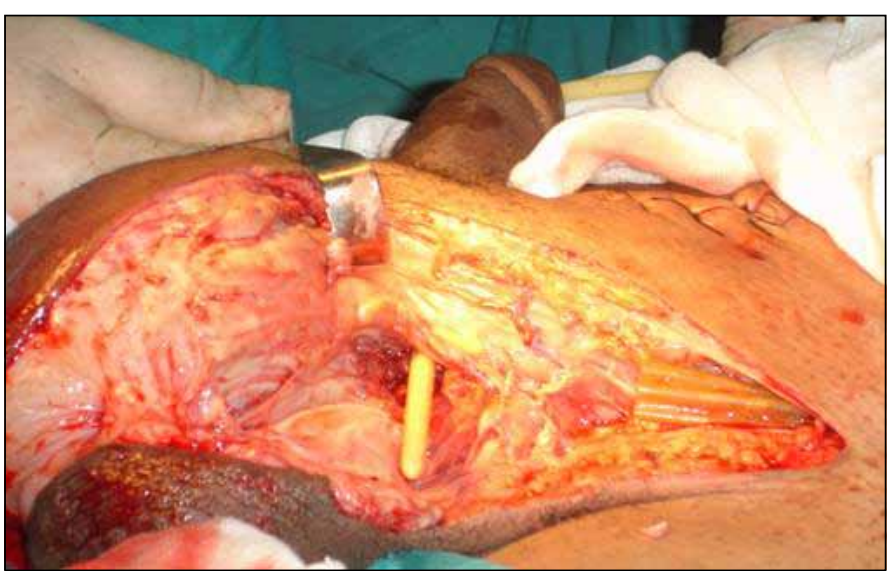

Fig. 3. Rupture de l'urètre transtumorale.

chaque fois qu'apparaît chez un patient suivi pour rétrécissement urétral un événement inhabituel tel que dilatation hémorragique, apparition d'une tuméfaction génitale, aggravation rapide de la dysurie, fistule ou écoulement urétral purulent.

Dans le cas qui nous concerne, on a noté une rupture spontanée de l'urètre transtumorale avec constitution d'un urinome pelvien témoignant d'une évolution locale défavorable. Il s'agit à la fois d'un événement exceptionnel de l'évolution de la tumeur et aussi d'une étiologie exceptionnelle de rupture de l'urètre par envahissement tumoral de la paroi urétrale et sa fragilisation. Le diagnostic a été posé après mise à plat de l'urinome infecté et la découverte peropératoire d'une tumeur de l'urètre bulbaire.

L'urétrographie permet d'évoquer le diagnostic en montrant des images caractéristiques comme c'est le cas chez notre patient, à savoir une lacune marécageuse et une rigidité segmentaire d'un des bords de l'urètre. L'urétrocystoscopie permet de faire des biopsies dont l'étude histologique est requise pour le diagnostic.

Sur le plan histologique, $80 \%$ de ces tumeurs sont des carcinomes épidermoïdes, $15 \%$, des carcinomes urothéliaux et $5 \%$, des adénocarcinomes. L'examen cytologique a une grande valeur lorsqu'il est positif ${ }^{10}$.

Le cancer de l'urètre masculin est une maladie à malignité locorégionale qui s'étend aux corps érectiles (caverneux ou spongieux) ou aux ganglions lymphatiques régionaux. Les adénomégalies inguinales sont retrouvées dans un tiers des cas. Les métastases ne s'observent que dans 10 à $20 \%$ des cas ${ }^{4}$.

Une fois le diagnostic posé et avant tout traitement, l'évaluation et la stadification de la tumeur reposent sur l'examen clinique sous anesthésie, l'urétrocystoscopie, la tomodensitométrie abdomino-pelvienne et l'imagerie par résonance magnétique (IRM). La TDM donne une bonne analyse des aires ganglionnaires et pelviennes ${ }^{13}$. Sa sensibilité et sa spécificité dans l'évaluation en profondeur des tumeurs de l'urètre ont fait proposer à certains l'étude

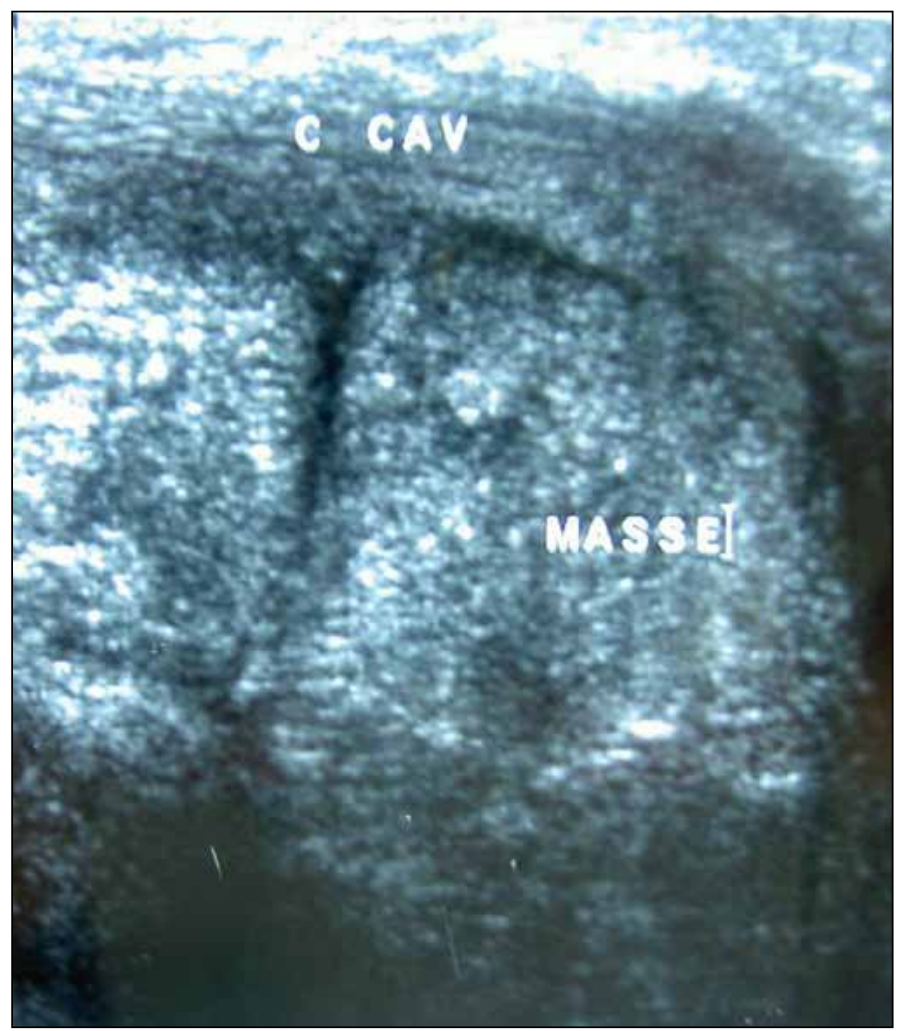

Fig. 4. Une échographie périnéale et endorectale du processus tissulaire de l'urètre membraneux étendu à la partie postérieure des corps caverneux avec absence d'extension vers le complexe vésico-prostatique.

du pénis par IRM. Ceci permet de mesurer l'étendue de la tumeur au-delà du corps spongieux car l'hyposignal correspondant à la ligne du fascia de l'albuginée sera interrompu au niveau de l'infiltration tumorale ${ }^{12}$. Certains proposent l'échographie comme examen de première intention pour l'évaluation locale de l'infiltration tumorale, en particulier pour les lésions de l'urètre antérieur ${ }^{14}$. En 1980, l'échographie par voie endorectale ou trans-scrotale a été utilisée pour évaluer les lésions de l'urètre postérieur, mais sans grand succès ${ }^{15}$. II n'existe pas à ce jour de série évaluant les tumeurs de l'urètre par échographie. Nous pensons que cet examen est particulièrement spécifique pour détecter I'envahissement des corps spongieux et caverneux mais sa faible sensibilité limite son utilisation.

Le traitement repose sur l'exérèse chirurgicale large dont l'étendue dépend de la localisation et du stade de la tumeur, sauf pour les rares cas de tumeurs superficielles (stade O ou A de Whitmore) qui peuvent bénéficier d'un traitement endoscopique (résection transurétrale, fulguration, laser YAG...).

Schématiquement, le traitement consiste en une urétrectomie segmentaire en cas de tumeur localisée et une pénectomie partielle ou une pénectomie radicale avec urétrostomie périnéale pour les lésions de l'urètre antérieur. Alors que les atteintes de l'urètre bulbo-membraneux sont discutables, une cystoprostatectomie avec pénectomie et urétrectomie, 


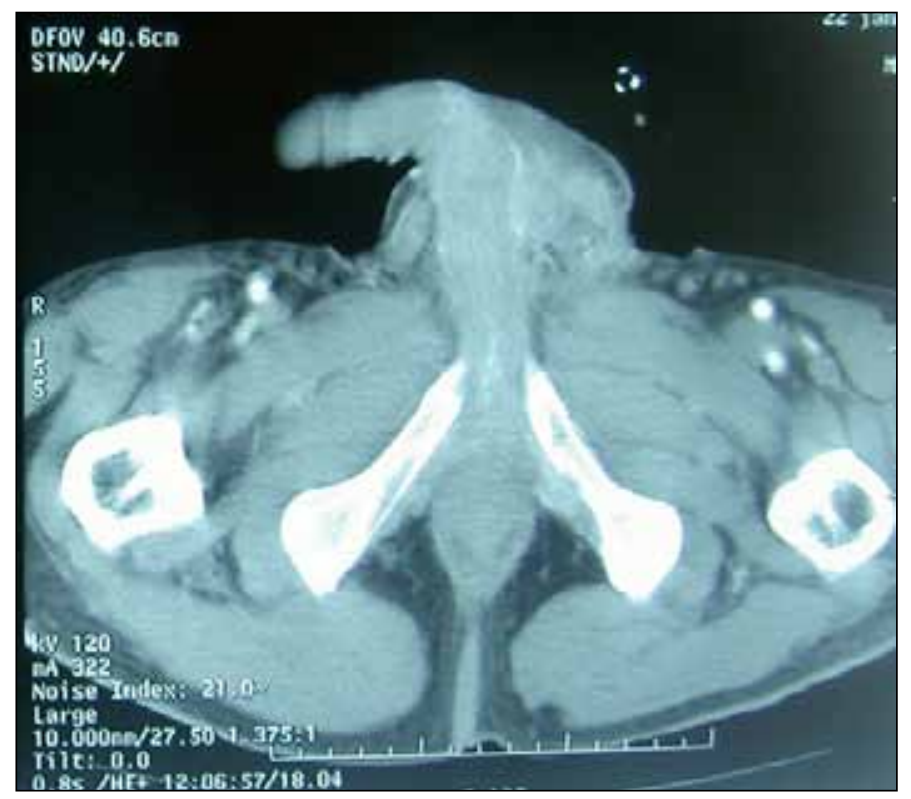

Fig. 5. TDM - Tumeur de l'urètre membraneux étendue à la partie postérieure des corps caverneux.

voire même émasculation et pubectomie pour les tumeurs de l'urètre postérieur, peut être indiquée $e^{16,17}$.

Le pronostic des lésions de l'urètre antérieur est meilleur que celui des lésions de l'urètre bulbo-membraneux.

Selon Dalbagni et ses collaborateurs, la survie après 5 ans est de $69 \%$ et $26 \%$, respectivement, pour les lésions de l'urètre antérieur et de l'urètre bulbo-membraneux ${ }^{17}$. Les tumeurs de l'urètre postérieur sont en général mises en évidence à un stade plus avancé que les tumeurs de l'urètre antérieur, car elles sont moins accessibles aux interventions diagnostiques et thérapeutiques et ont par conséquent un plus mauvais pronostic ${ }^{8}$.

La radiothérapie est considérée comme décevante, surtout pour les lésions de l'urètre bulbo-membraneux. Kaplan et ses collaborateurs ${ }^{4}$ ont rapporté que sur 186 cas colligés dans la littérature, on retrouvait 11 cas traités par radiothérapie seulement; seuls deux patients avaient eu une survie supérieure à 5 ans.

La chimiothérapie a pu donner de bons résultats dans les stades avancés, en complément d'un traitement chirurgical mutilant afin d'obtenir le contrôle local de la maladie ${ }^{18}$.

Cependant, l'espoir actuel chez les malades porteurs $d^{\prime}$ 'un carcinome épidermoïde de l'urètre réside dans des protocoles thérapeutiques combinant radiothérapie et chimiothérapie en se basant sur les résultats obtenus dans les cancers épidermoïdes de l'œesophage et de l'anus ${ }^{19}$, ce qui permettrait une chirurgie moins mutilante. II est toutefois difficile d'extrapoler ces résultats aux tumeurs de l'urètre.

Ainsi, le traitement de référence pour les stades avancés semble néanmoins être l'association chimiothérapie, radiothérapie externe et résection chirurgicale extensive. La chimiothérapie utilise surtout le 5-FU et le cisplatine ou la mitomicyne ${ }^{8}$. La radiothérapie externe emploie des doses de 45 Gy sur le pelvis (incluant les aires ganglionnaires si elles sont cliniquement atteintes) et une irradiation supplémentaire de 25 à 30 Gy sur la lésion primaire ${ }^{8}$. La chimiothérapie associée à la radiothérapie agit comme agent radiosensibilisant en interférant avec les processus de réparation cellulaire après irradiation ${ }^{12}$. II semble en particulier que le 5-FU couplé à la radiothérapie engendre un effet antinéoplasique synergique ${ }^{20}$.

\section{Conclusion}

La rupture spontanée de l'urètre transtumorale est un mode de découverte exceptionnel témoignant d'une évolution locale défavorable, ce qui rend ces tumeurs difficilement opérables. La rareté de la lésion et le faible nombre de cas rapportés font qu'il est difficile d'harmoniser les attitudes thérapeutiques vis-à-vis de la tumeur. Cependant, I'espoir actuel réside dans des protocoles thérapeutiques combinant radiothérapie et chimiothérapie.

\section{Bibliographie}

1. Nishimura K, Kameoka H, Kondoh M, et al. Mucinous adenocarcinoma of the male urethra. Urol Int 1996;57:246-8.

2. Tefilli MV, Gheiler EL, Shekarriz B, et al. Primary adenocarcinoma of the urethra with metastasis to the glans penis: successful treatment with chemotherapy and radiotherapy. Urology 1998;52:517-9.

3. Hopkins SC, Nag SK, Soloway MS. Primary carcinoma of male urethra. Urology 1984;23:128-33.

4. Kaplan GW, Bulkley GJ, Grayhack JT. Carcinoma of the male urethra. J Urol 1967;98:365-9.

5. Wiener JS, Liu Walther PJ. Oncogenic human papillomavirus Type 16 is associated with squamous cell cancer of the male urethra. Cancer Res 1992:52:5018-23.

6. Van de Voorde W, Meertens B, Baert L, et al. Urethral squamous cell carcinoma associated with urethral stricture and urethroplasty. Eur I Surg Oncol 1994;20:478-83.

7. Perez CA, Pilepich MV. Penis and male urethra. Principles and practice of radiation oncology 1992; chapter 53:1135-42.

8. Gheiler EL, Tefilli MV, Tiguert R, et al. Management of primary urethral cancer. Urology 1998;52:487-93.

9. Boulduan JP, Farah N. Primary urethral neoplasms: review of 30 cases. J Urol 1981;125:198-200

10. Hotchkiss RS, Amelar RD. Primary carcinoma of the male urethra. J Urol 1954;72:1181-91.

11. Vapnek JM, Hricak H, Carroll PR. Recent advances in imaging studies for staging of penile and urethral carcinoma. Urol Clin North Am 1992;19:257-66.

12. Helenon 0 , Hamida K, Augusti $M$, et al. Radiologie de l'urètre pathologique. EMC (Elsevier, Paris). Radiodiagnostic - Urologie - Gynécologie, 34-410-A20, 1992, 18p.

13. Fair WR, Fuks ZY, Schmer MI. Cancer of the urethra and penis. In : De Vita VT, Hellman S, Rosenberg SA eds. Principles and fracture of oncology. 4th edition. Philadelphia, PA: Lippincott Company; 1993:1114-25.

14. Klosterman PW, Laing FC, Mcaninch JW. Sonourethrography in the evaluation of urethral stricture disease. Urol Clin North Am 1989;16:791-7.

15. Mcaninch JW, Laing FC, Jeffrey RB. Sonourethrography in the evaluation of urethral strictures : a preliminary report. J Urol 1988;139:294-7.

16. Farrer JH, Lupu AN. Carcinoma of the deep male urethra. Urology 1984;24:527-31.

17. Dalbagni $G$, Zhang ZF, Lacombe $L$, et al. Male urethral carcinoma: analysis of treatment outcome. Urology 1999;53:1126-32

18. Baskin LS, Turzan C. Carcinoma of male urethra: management of locally advanced disease with combined chemotherapy, radiotherapy and penile-preserving surgery. Urology 1992;39:21-5.

19. Coia LR, Engstrom PF, Paul A. Non surgical management of urethral cancer: report of a study of combined radiotherapy and radiotherapy and chemotherapy. J Clin Oncol 1987;5:1783-7.

20. Yagoda A. Chemotherapy of urothelial tract cancer: Memorial Sloan- Kettering Cancer Center experience. Important Adv Oncol 1988;4:143-59.

Correspondence: Dr. Jilani Ghorbel, Service d'urologie, Hôpital Charles Nicolle, Tunis, Tunisie ; ghorbelijlani@yahoo.fr 